\title{
PROTEINS, PHOTOSYNTHETIC PIGMENTS AND POLAR LIPIDS CHANGES OF THYLAKOID BIOMEMBRANE FROM PRUNUS AFFECTED BY IRON CHLOROSIS.
}

\section{E.Monge; L. Montañés and J. Val.}

Estación Experimental Aula Dei. Apt 202. 50080-Zaragoza.

\section{Spain.}

\section{$\underline{\text { Abstract }}$}

The effect of iron chlorosis on proteins, photosynthetic pigments and acyl lipid composition of adult field grown peach tree leaves has been studied. No differences in the samples have been found between the iron extracted by $\alpha, \alpha^{\prime}$ - dipyridyl (active iron) and total iron analysed by atomic absorption spectrometry, both analyses are good determinations to detect iron chlorosis. The levels of proteins, photosynthetic pigments and acyl lipids were reduced under iron chlorosis, although the concentrations of proteins and polar lipids per chlorophyll unit were higher in the chlorotic peach tree than in the control.

Key Index Words: active iron, total iron, photosynthetic pigments, VAZ cycle, polar lipids, thylakoid protein, peach tree.

\section{Introduction}

Iron-chlorosis is a big problem, mainly in arid and semi-arid regions, whose soils contain high concentrations of calcium carbonate.

Although iron is abundant in calcareous soils, usually it is not usually available for fruit trees, including peach trees. The leaves from iron deficient plants, can or cannot have a total iron similar to control plants and this problem could indicate a physiological inactivation of some fraction (non-physiological iron) of the inside iron.

Iron deficient plants show visible symptoms in young leaves, because their chlorophyll contents decline and their colour becomes green-yellow (chlorotic). 
The effects of iron deficiency mainly take place inside chloroplasts, while the rest of cell organelles are not usually affected. The iron deficiency preferentially inhibits the development of the photosynthetic membranes of the chloroplats, with few grana stakes.

The purpose of this study is to analyse the effects of iron deficiency on the composition of their chloroplast membrane components: proteins, photosynthetic pigments, galactolipids and phospholipids.

\section{Material and methods}

Leaves (about 30-40) from the middle part of the shoot were harvested in July, around the canopy from adult peach trees (prunus persica L. cv. Miraflores) grown on calcareous soils (approximated pH 8) in Zaragoza (Spain).

To determine the total iron, the leaves, with their petioles removed, were washed to eliminate surface contamination. Dry ashing was carried out following the method of C.I.I. (1969) and Pinta and DeWele (1975). The iron was determined by atomic absorption spectrometry. The determination of the physiological iron was carried out following the method of Abadía et al. (1984), using $\alpha$ - $\alpha^{6}$-dipyridyl.

Photosynthetic pigments were extracted from leaf disks, which were ground with 5 $\mathrm{ml}$ of $100 \%$ acetone and a few mg of sodium ascorbate. The quantitative determinations were carried out following the method of Val et al. (1994) by HPLC.

Thylakoid polar acyl lipids were extracted from leaves, according to the method described by Bligh and Dyer (1959). The analysis of the different lipids by TLC was carried out following the method of Trémolières and Lepage (1971).

\section{Results}

Figure 1 shows concentrations of active iron and total iron according to their total chlorophyll. The lineal distribution between total iron and total chlorophyll answers to equation $\mathrm{y}=2.32 \mathrm{X}+50.95$ and its correlation coefficient was $\mathrm{R}^{2}=0.903$. On the other hand, the lineal distribution between total iron and total chlorophyll was $\mathrm{y}=$ $0.058 x+1.274$ and its correlation coefficient was $\mathrm{R}^{2}=0.903$. 


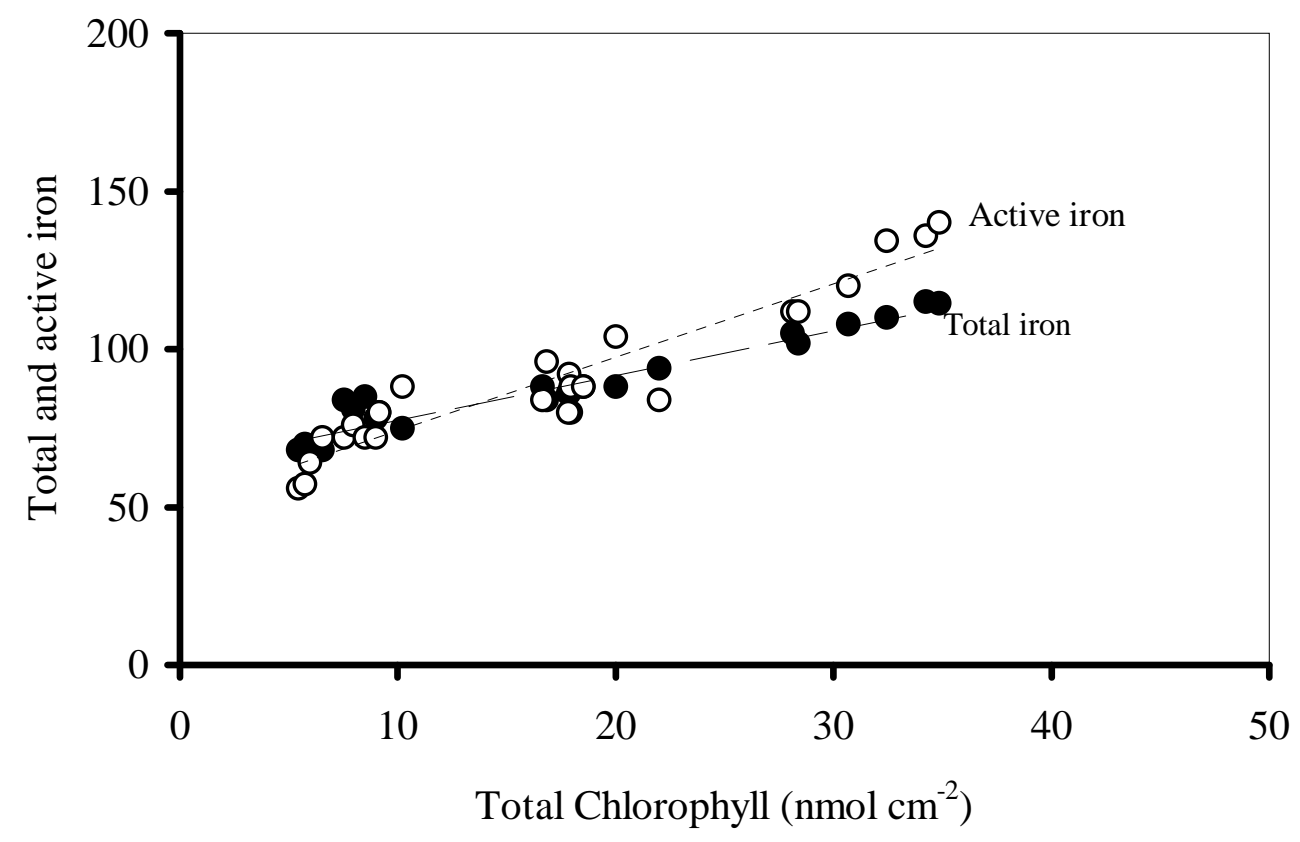

Fig 1 .- Regression lines of total and active (multiplied by 40) iron according to total clorophyll in leaves.

The active iron concentrations have been multiplied by 40 to put them inside total iron range and so both values can be showed in the figure 1 .

Both correlation coefficients (total and active iron) are very heigh, which proves that both, the total iron and the active iron, are strongly correlated with total chlorophyll and that the chlorosis of the leaves harvested is caused by iron deficiency.

Figure 2 shows the thylakoid protein concentration per chlorophyll unit according to total chlorophyll. In this figure it is shown that the amount of thylakoid protein per unit of total chlorophyll is bigger according to the increase in the grade of iron chlorosis of peach tree leaves. 


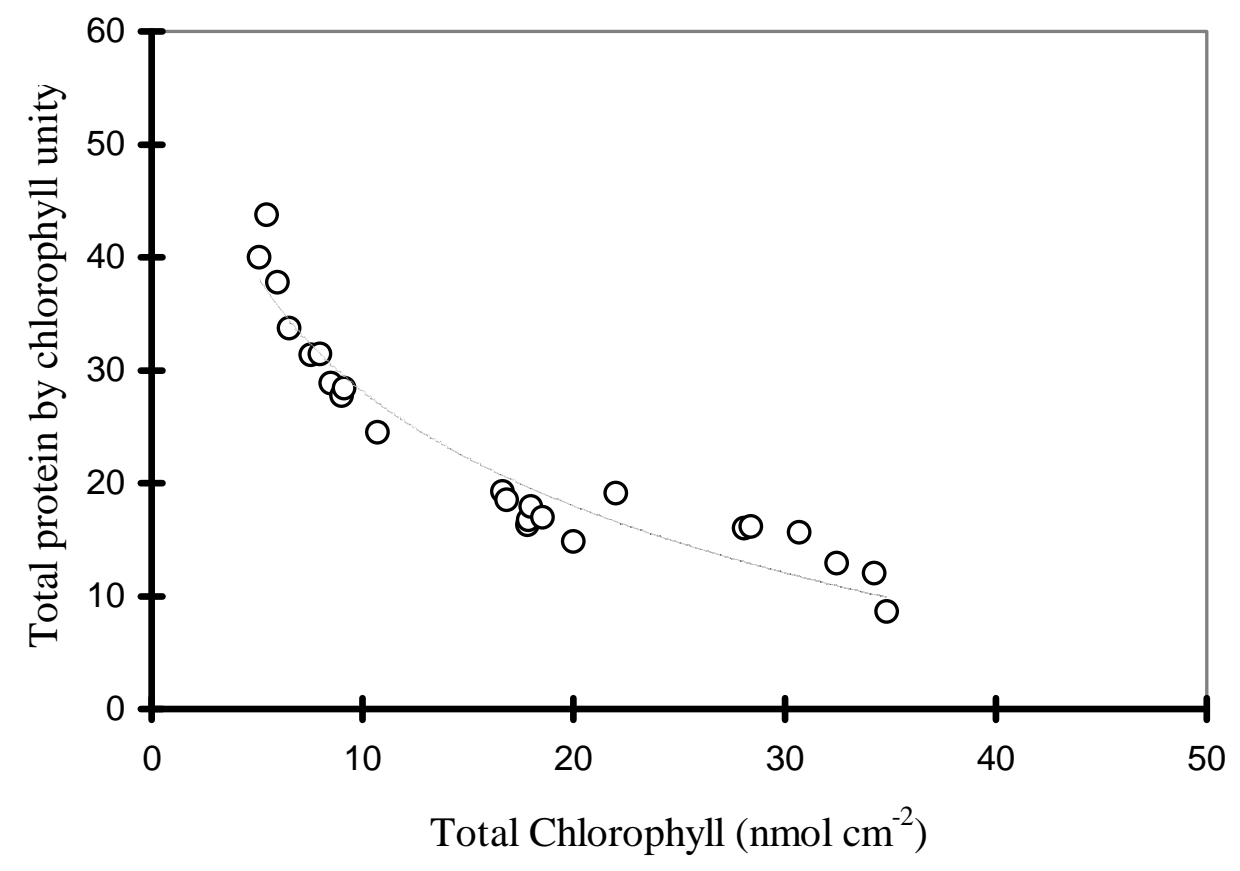

Fig. 2.- Lineal evolution of total protein by chlorophyll unity according to level of total chlorophyll

The distribution between thylakoid protein and total chlorophyll answered better to an evolution of the logarithmic type and its equation was $y=14.59 \operatorname{Ln}(\mathrm{X})+61.75$ and its correlation coefficient $\mathrm{R}^{2}=0.923$. These results show that the quantity of thylakoid protein per unit of chlorophyll is smaller in non-deficient peach tree leaves.

The photosynthetic pigments were analysed by HPLC. In figure 1 the relationship between both kind of iron and the chlorophyll has already been shown and hence the lack of iron in leaves (total and/or active) causes a lack of chlorophyll in them. In figure 3 , the evolution (in percentage) of the three photosynthetic pigment components of the VAZ cycle is shown. The study of the results achieved by HPLC have shown that iron deficiency also reduced the leaf concentrations of neoxanthin, luthein (other xanthophyll) and the $\beta$-carotene (results not shown). 


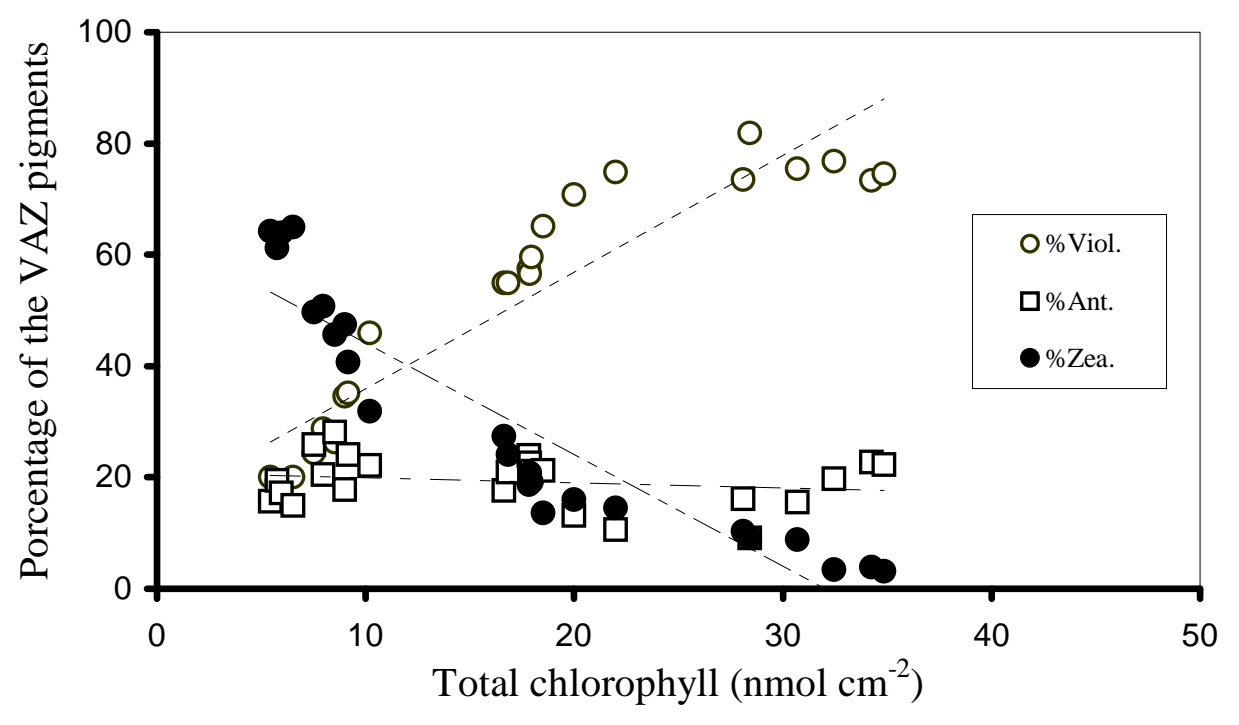

Fig. 3.- Lineal evolutions of the Violaxanthin, Anteraxanthin and Zeaxanthin (VAZ pigments) according to total chlorophyll.

The lineal distribution of the VAZ pigments as well as their correlation coefficients are given in table 1.

Table 1.- Equations of the lineal distribution and their respective correlation coefficients for pigments of the VAZ cycle.

\begin{tabular}{|l|c|l|}
\hline VAZ pigment & Equation & Correlation coefficient \\
\hline Violaxanthin & $\mathrm{y}=2.099 \mathrm{X}+14.90$ & $\mathrm{R}^{2}=0.864$ \\
\hline Antheraxanthin & $\mathrm{y}=-0.096 \mathrm{X}+20.94$ & $\mathrm{R}^{2}=0.042$ \\
\hline Zeaxanthin & $\mathrm{y}=-2.003 \mathrm{X}+64.16$ & $\mathrm{R}^{2}=0.858$ \\
\hline
\end{tabular}

These results show that although the antheraxanthin is not well correlated with total chlorophyll, both violaxanthin and zeaxanthin have very high correlations, and while concentrations of violaxanthin are high in non stressed (chlorotic) plants, the concentrations of zeaxanthin are very low or not considerable in the chromatogram.

The polar lipids were extracted from thylakoids in methanol-chloroform-water (1:1:1) according to the method mentioned above (material and methods). After 
extraction, the polar lipids and also the photosynthetic pigments remained in the chloroform phase. The concentration of total chlorophyll, in this phase, was also analysed and, in this way, the concentrations of polar lipids could be expressed per total chlorophyll unit.

In figure $4 \mathrm{~A}$ the lineal regression between total acyl lipids per chlorophyll unit versus the concentration of chlorophyll in leaves is shown. The better kind of regression curve found to express this relation has been logarithmic and its equation was $\mathrm{y}=$ $2.907 \operatorname{Ln}(\mathrm{X})+11.46$, the correlation coefficient being $\mathrm{R}^{2}=0.938$. The negative expression of this equation shows that the concentration of polar lipids per unit of chlorophyll is higher in chlorotic plants (according to total chlorophyll) than in non-

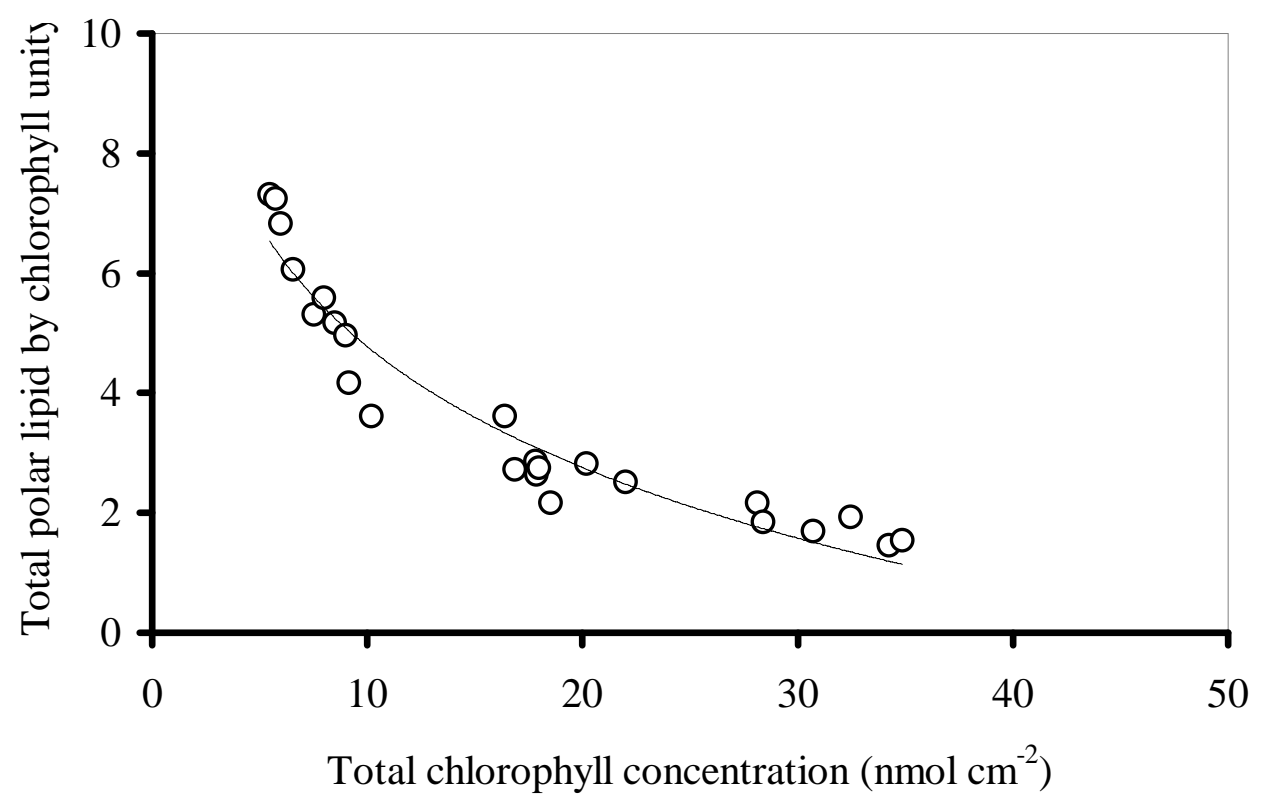

stressed plants.

Fig 4 A.- Evolution of the ratio of total polar lipids by chlorophyll unity according to total chlorophyll in thylakoid.

The analysis of the lipid extracts obtained and analysed by TLC revealed the existence of several kinds of lipids: MGDG (monogalactosyldiglycerol), DGDG (digalactosyldiglycerol), PC (phophatidylcholine) and PG (phophatidylglycerol). 
The study of the these results (not shown) has proved that while the concentration of the total galactolipids decreased according to the increase in the degree of chlorosis in leaves, the total phospholipids increase in the same proportion.

The analysis of the two galactolipids components has proved that the decrease of the total galactolipids was due to a slight decrease of both components. The decrease of the MGDG was also accompanied by a reduction of the DGDG (Fig. 4 B).

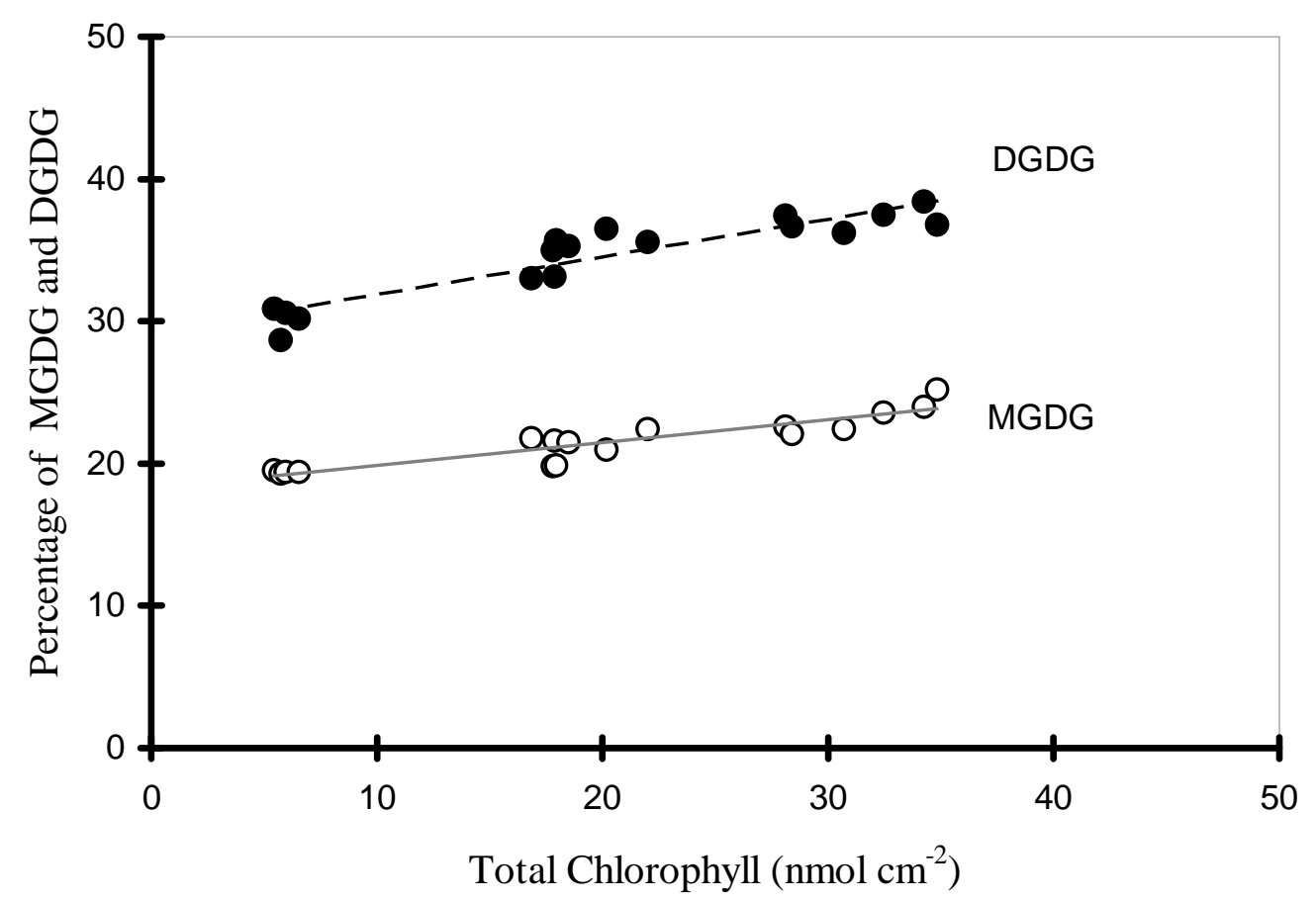

Fig. 4B.- Lineal evolution of the percentage of major galactolipids according to total chlorophyll concentrations.

The iron deficiency also alters the phospholipid levels. The percentage of total phospholipids increased with low concentrations of chlorophyll. In figure $4 \mathrm{C}$ the lineal evolution of two major components phospholipids is shown. While the percentage of PC increases with the iron deficiency, the percentage of PG decreases slightly according to the decrease in total chlorophyll in peach tree leaves. 


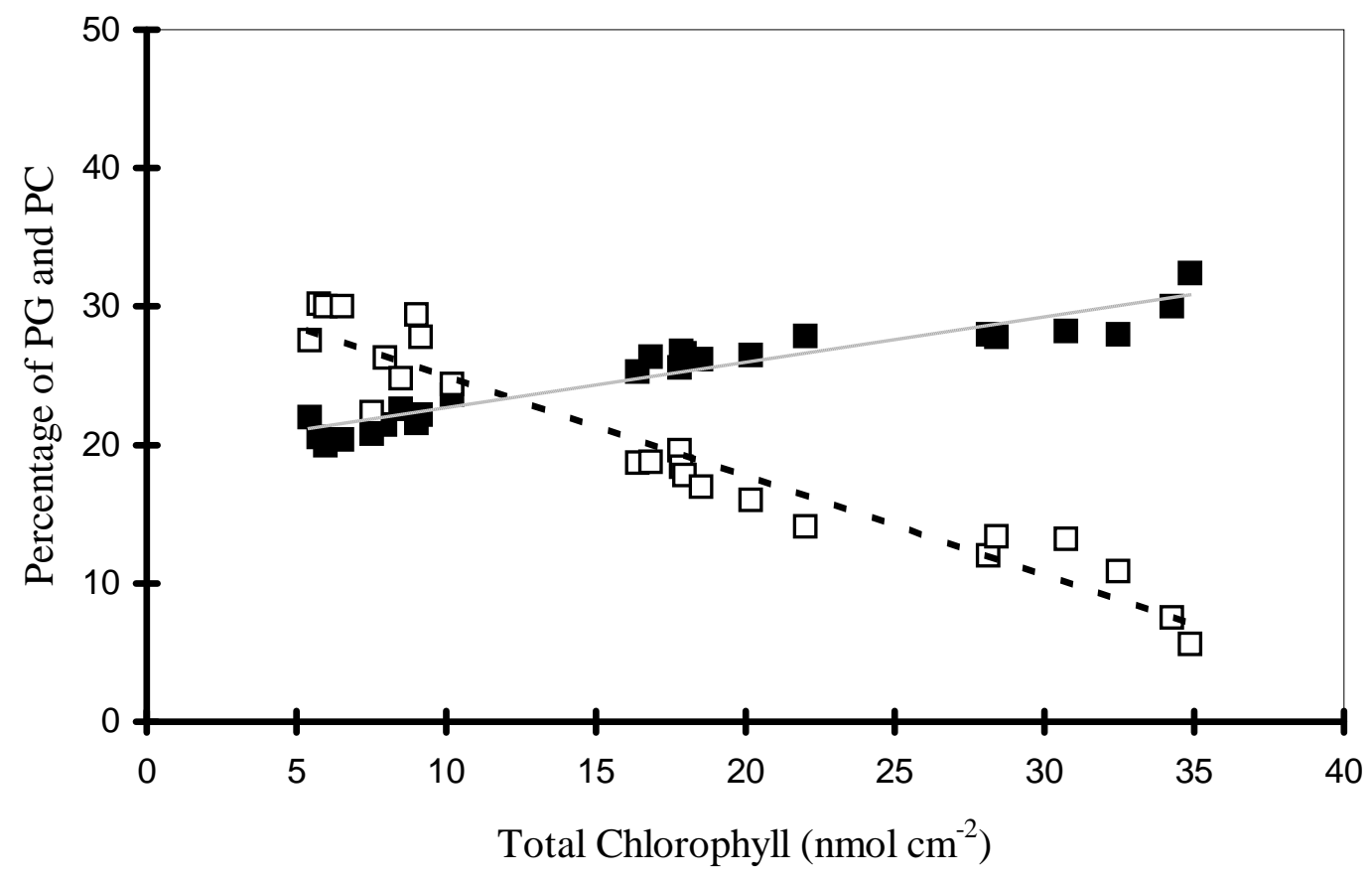

Fig. 4 C.- Lineal evolution of the percentage of major phospholipids according to total chlorophyll concentrations.

The equations of the both components of galactolipids and phospholipids and the correlation coefficients are specified in table 2. While the MGDG and DGDG have not shown any significant correlation with the total chlorophyll, the PG and PC have shown a good correlation with this pigment.

Table 2.- Equations of the lineal distribution and their respective correlation coefficients for pigments of the major acyl lipid components

\begin{tabular}{|c|l|c|c|}
\hline \multicolumn{1}{|c|}{ Acyl lipids } & Components & Equation & $\mathbf{R}^{2}$ \\
\hline \multirow{2}{*}{ Galactolipids } & MGDG & $\mathrm{y}=0266 \mathrm{X}+29.18$ & 0.853 \\
\cline { 2 - 4 } & DGDG & $\mathrm{y}=0.161 \mathrm{X}+18.44$ & 0.840 \\
\hline \hline \multirow{2}{*}{ Phospholipids } & PG & $\mathrm{y}=0.328 \mathrm{X}+19.39$ & 0.903 \\
\cline { 2 - 4 } & PC & $\mathrm{y}=-0.724 \mathrm{X}+32.26$ & 0.926 \\
\hline
\end{tabular}




\section{Discussion}

The iron chlorosis manifests itself on the young leaves on the new shoots. The intervenal space of the leaves starts to become yellow (Bindra, 1980).

In our study, the two kinds of iron analysed in leaves was affected by iron deficiency. Both the total and active irons were lower in chlorotic than control leaves and as the total iron is always bigger than the active iron, this would show that a fraction of the total iron is not physiologically active, although its metabolic role is not well known.

The iron chlorosis also caused a reduction in the proteins of the thylakoid membranes, although the level of protein per chlorophyll unit is bigger in chlorotic samples. These data are in agreement with the results shown by Nishio and Terry, 1983.

The high correlation found between both total and active iron and total chlorophyll proves that the lack of this pigment was only induced by iron-deficiency. Monge et al., 1987 demonstrated that iron deficiency affects all photosynthetic pigments, but more drastically to chlorophylls ( $\mathrm{a}$ and $\mathrm{b}$ ) and the $\beta$-carotene, being the xanthophyll group, especially the luthein, decreased the least. As the chlorotic plants are more sensitive to photoinhibitory conditions (Thompson 1987), where the VAZ cycle plays an important role in dissipating the excess of the excitation energy that reaches the photosynthetic apparatus. In figure 3 it can be observed that the level of zeaxanthin is increased at the expense of the violaxanthin, in chlorotic samples, the antheraxanthin does not seem to be affected by iron deficiency.

According to Platt-Aloia et al., 1983, iron chlorosis causes a reduction in the staking organisation of the thylakoid membrane system of chloroplasts and probably this lack of staking could be the cause of changes in the acyl lipid composition, a decrease of galactolipids and an increase in phospholipids. On the other hand, it has been shown that the levels of total polar lipid per chlorophyll unit are higher in chlorotic samples that in non-deficient plants, but these results are very similar to those obtained by proteins.

The whole study of these results, together with others published in previous papers (Monge et al., 1987 and 1993), show that the lack of iron in plants affects all 
components of the thylakoid membranes (proteins, photosynthetic pigments, acyl lipids), but in different proportions. The photosynthetic pigments are the components most affected, chlorophyll being the most sensitive, after the xantophyll group, and although the proteins and acyl lipids are also affected, they are less affected by iron chlorosis.

\section{Acknowledgements}

The authors wish to thank $\mathrm{M}^{\mathrm{a}}$. A. Gracia for her excellent technical assistance

\section{References}

Abadía J., Monge E., Montañés L. and Heras L. 1984. Extraction of iron from plant leaves by Fe (II) chelators. J. Plant Nutr. 7: 777-784.

Bindra A S 1980 Iron chlorosis in horticultural and field crops. In Annual Review of Plant Sciences. Eds. C. P. Malik. Vol. II. pp 221-312. Kalyani, New Delhi, India.

Bligh E. and Dyer W. J. 1959. A rapid method of total lipid extraction and purification. Can. J. Biochem. 37: 911-917.

C.I.I. 1969 Comité Inter-Institutos para el estudio de técnicas analíticas. Métodos de referencia para la determinación de elementos minerales en vegetales. An. Edaf. Agrobiol. 38: 403-417

Lowry O.H. Rosebrough N.J. Farr A.L. and Randall R.J. 1951. Protein measurement with the folin phenol reagent. J. Biol. Chem. 193: 265-275.

Monge E Val J Heras L and Abadía J 1987 Photosynthetic pigment composition of higher plants grown under iron stress. Progress Photosyn. Res. 4, 201-204.

Monge, E., Pérez, A., Pequerul, A, Madero, A and Val, J. 1993. Effect of iron on mineral nutrition and lipid composition of thylakoid in Prunus persica (L.) Bastch. Plant and Soil 154:97-102.

Nishio J and Terry N 1983 Iron nutrition mediated chloroplast development. Plant Physiol. 71, 688-691.

Pinta M and DeWele G 1975 Etalons végétaux pour l'analyse foliaire. In Le côntrole de l'alimentation des plantes cultivées. Eds. K.Pozman Vol. pp 159-172. Akademiai Kiado, Budapest.

Platt-Aloia , K.A., Thompson W.W. and Terry N. 1983. Changes in plastid ultrastructure during iron nutrition mediated chloroplast development. Protoplama 114: 85-92.

Thompson L K 1987 Irradiation stress. In: The physiology of plants under stress. (MG Hale DM Orcutt eds.) Wiley-Interscience. New York. pp 103-116.

Trémolières A. and Lepage M.1971. Changes in lipid composition during greening of etiolated pea seedlings. Plant Physiol. 37: 911-917.

Val J., Monge E. and Baker N.R. 1994. An improved HPLC method for rapid analysis of the xanthopyll cucle pigments. J. Chromatogr. Sci. 32: 286-289. 